Bull. Austral. Math. Soc.

$55 \mathrm{R} 10,57 \mathrm{R} 30$

VOL. 37 (1988) [173-177]

\title{
STRUCTURE PRESQUE-TRANSVERSE INTÉGRABLE
}

\section{TONG VAN DUC}

\begin{abstract}
We find conditions for which a manifold endowed with an integrable almost transversal structure is the total space of a vector bundle isomorphic to the transversal bundle of a foliation.
\end{abstract}

Les variétés considérées sont connexes et paracompactes. Tous les éléments introduits sont de classe $C^{\infty}$.

DÉfINITION. Une structure presque-transverse sur une variété différentiable est une $G$-structure dont les éléments de $G$ sont de la forme :

$$
\left[\begin{array}{lll}
A & B & 0 \\
0 & C & 0 \\
0 & D & C
\end{array}\right]
$$

où $A \in G l(p, \mathbb{R})$ et $C \in G l(q, \mathbb{R})$.

ExEmple: Soit $M$ une variété différentiable de dimension $p+q$ muni d'un feuilletage $\mathcal{F}$ de codimension $q . \quad M$ est défini par un atlas $\mathcal{A}=\left\{U, x^{u}, x^{a}\right\}$ dont les fonctions de transition vérifient :

$$
\frac{\partial x^{\prime a}}{\partial x^{u}}=0
$$

où $u, v, \cdots=1 \ldots, p$ et $a, b, \cdots=1 \ldots, q$.

Soit $(Q, p, M)$ le fibré transverse du feuilletage : c'est le quotient de $T M$ par le fibré vectoriel des vecteurs tangents aux feuilles de $\mathcal{F}$. Chaque élément $\bar{X}$ de $Q$ a pour représentant un vecteur $X$ de la forme :

$$
X=X^{u} \frac{\partial}{\partial x^{u}}+X^{a} \frac{\partial}{\partial x^{a}} .
$$

Comme $X^{a}$ ne dépend que de $\bar{X}$, on prendra $\left(x^{u}, x^{a}, X^{a}\right)$ comme coordonnées locales dans $p^{-1}(U)$. On obtient ainsi un atlas de $Q$ dont les fonctions de transition sont de la forme :

$$
x^{\prime u}=x^{\prime u}\left(x^{u}, x^{a}\right), x^{\prime a}=x^{\prime a}\left(x^{a}\right), X^{\prime a}=\frac{\partial x^{\prime a}}{\partial x^{b}} X^{b} .
$$

Received 31 March 1987

Copyright Clearance Centre, Inc. Serial-fee code: 0004-9729/88 \$A2.00+0.00. 
Les matrices jacobiennes de ces transformations sont du type (1). Ainsi la variété $Q$ est munie d'une structure presque-transverse intégrable.

On se propose de chercher dans quelles conditions une variété munie d'une structure presque-transverse intégrable peut être considérée comme l'espace total du fibrétransverse d'un feuilletage.

Si une variété $N$ est munie d'une structure presque-transverse, il existe sur $N$ une structure presque-tangente $J$ dont l'expression locale dans un repère mobile $\left(e_{u}, e_{a}, e_{a^{*}}\right) .\left(a_{*}, b_{*}, \cdots=1 \ldots, q\right)$ est la matrice

$$
\left[\begin{array}{lll}
0 & 0 & 0 \\
0 & 0 & 0 \\
0 & I & 0
\end{array}\right] .
$$

Cette structure presque-tangente joue un rôle important dans la suite.

D'autre part, il existe sur $N$ une distribution $\tilde{\mathcal{F}}$ de dimension $p$ dont une base locale est $\left(e_{u}\right)$. L'image de $J$ est une distribution $\mathcal{L}$ de dimension $q$ engendrée par $\left(e_{a *}\right)$.

Si la structure presque-transverse est intégrable, la structure presque-tangente est intégrable. Ceci est équivalent au fait que la torsion de Nijenhuis $N_{J}$ de $J$ est nulle, c'est à dire

$$
N_{J}(X, Y)=[J X, J Y]-J[J X, Y]-J[X, J Y]=0
$$

$\forall X, Y \in \mathcal{X}(M)$. De plus les distributions $\mathcal{L}$ et $\tilde{\mathcal{F}}$ sont intégrables. On désigne encore par $\mathcal{L}$ et $\widetilde{\mathcal{F}}$ les feuilletages correspondants.

EXEMPlE: La variété $N=\mathbf{R}^{p} \times \mathrm{T}^{2}$ où $\mathrm{T}^{2}$ est le tore de dimension 2 est munie d'une structure presque-transverse intégrable. Si l'on désigne par $\left(t^{u}, x, y\right)$ les coordomnées locales de $\mathrm{R}^{p} \times \mathrm{T}^{2}$, la distribution $\mathcal{L}$ est engendrée par le champ $\frac{\theta}{\partial y}$ et on a une submersion $\pi: N \rightarrow M=N / \mathcal{L}=\mathbf{R}^{p} \times S^{1}$, dont les fibres sont des cercles. Ainsi $N$ ne peut être l'espace total d'un fibré transverse d'un feuilletage.

On rappelle la définition et les propriétés d'un fibré affine qui sont utiles pour la suite.

DÉfinition. Soit $\pi: A \rightarrow M$ une submersion surjective. Le triplet $(A, \pi, M)$ est un fibré affine modelé sur un fibré vectoriel $(E, p, M)$ s'il existe une application différentiable $\rho: A \times_{M} E \rightarrow A$ telle que $\forall x \in M, \rho_{x}: A_{x} \times E_{x} \rightarrow A_{x}$ définisse sur $A_{x}$ une structure d'espace affine modelé sur $E_{x}$.

Proposition 1. [5] Tout fibré affine est un fibré localement trivial dont les fonctions de transition sont des applications affines. Tout fibré affine admet une section globale. 
On considère désormais une variété $N$ munie d'une structure presque-transverse intégrable. On suppose en plus que l'espace quotient $M=N / \mathcal{L}$ est une variété différentiable. Alors le feuilletage $\widetilde{\mathcal{F}}$ passe au quotient en sorte qu'on ait une submersion surjective $\pi$ de $N$ sur $M$ et que la restriction de $\pi$ à chaque feuille de $\tilde{\mathcal{F}}$ soit un difféomorphisme local. On note $\mathcal{F}$ le feuilletage induit sur $M$ et $(Q, p, M)$ le fibré normal de $\mathcal{F}$. Les vecteurs tangents aux fibrés de $\pi$ seront appelés vecteurs verticaux.

Soit $u \in Q_{x}$. On définit le relèvement vertical $u^{v}$ de $u$ et un point $y \in \pi^{-1}(x)$ de la façon suivante. Soit $X \in T_{x} M$ tel que $\bar{X}=u$ et $A \in T_{y} N$ tel que $\pi_{*}(A)=X$. Alors

$$
u^{v}=J(A)
$$

$u^{v}$ est bien défini car si $X^{\prime} \in T_{x} M$ est tel que $u=X^{\prime}$ et $A^{\prime} \in T_{y} N$ tel que $\pi_{*}\left(A^{\prime}\right)=$ $X^{\prime}$, on a $X^{\prime}-X=Y \in \mathcal{F}_{x}$ et il existe $B \in \widetilde{\mathcal{F}}_{y}$ tel que $\pi_{*}(B)=Y$. Par suite, $\pi_{*}\left(A^{\prime}-A-B\right)=0$. Il en résulte que $J\left(A^{\prime}\right)=J(A)$ puisque $J(B)=0$. L'application $: u \rightarrow u^{v}$ de $Q_{x}$ sur l'espace tangente en $y$ à $\pi^{-1}(x)$ est un isomorphisme. Si $s=\bar{X}$ est une section de $Q$ et si $A$ est un champ de vecteurs sur $N$ tel que $\pi_{*}(A)=X$, $s^{v}=J(A)$ sera appelé relèvement vertical de $s$. On remarque que les relèvements verticaux des sections de $Q$ engendrent le module des champs verticaux de $N$.

Proposition 2. $\forall s, t \in \underline{Q}$, on a :

$$
\left[s^{v}, t^{v}\right]=0, L_{s^{v}} J=0 .
$$

Preuve: Soient $X, Y \in \mathcal{X}(M)$ tels que $s=\bar{X}$ et $t=\bar{Y}$ et soient $A, B \in \mathcal{X}(N)$ tels que $\pi_{*}(A)=X$ et $\pi_{*}(B)=Y$. On a :

$$
\left[s^{v}, t^{v}\right]=[J A, J B]=J[J A, B]+J[A, J B]=0
$$

car les champs $[J A, B]$ et $[A, J B]$ sont projetables et verticaux. $\forall C \in \mathcal{X}(N)$, $L_{s^{v}} J\left(C^{\prime}\right)=\left[s^{v}, J C\right]-J\left[s^{v}, C\right]$. Si $C=t^{v}$, on a $L_{v^{v}} J(C)=0$ comme on vient de le voir. Si $C^{\prime}$ est projetable, on a encore $L_{s} v J(C)=0$ pour les mêmes raisons.

Puisque la structure presque-transverse est intégrable, il existe sur $N$ une connexion sans torsion $\nabla$. Dans une carte $\left(U, x^{u}, x^{a}, x^{a_{*}}\right)$ adaptée à la $G$-structure, $\nabla$ est définie par :

$$
\nabla \frac{\partial}{\partial x^{i}} \frac{\partial}{\partial x^{j}}=0
$$

$(i, j, \cdots=1 \ldots, p+2 q)$. De plus, on a :

$$
\nabla J=0, \nabla \underline{\tilde{\mathcal{F}}} \subset \underline{\tilde{\mathcal{F}}}
$$

où $\underline{\mathcal{F}}$ désigne le module des sections de $\tilde{\mathcal{F}}$. 
Proposition 3. La connexion $\nabla$ induit sur chaque fibre de la submersion $\pi$ : $N \rightarrow M$ une connexion plate.

Preuve: -Soit $s, t \in \underline{Q}$. Utilisant les notations dans la preuve de la proposition 2, on obtient, puisque $\nabla J=0$ :

$$
\begin{aligned}
\nabla_{s^{v}} t^{v} & =\nabla_{s^{v}}(J B)=J\left(\nabla_{s^{v}} B\right) \\
& =J\left(\nabla_{B} s^{v}+\left[s^{v}, B\right]\right)=\nabla_{B}\left(J_{s^{v}}\right)+J\left[s^{v}, B\right]=0 .
\end{aligned}
$$

Il en résulte que la courbure de la connexion induite sur chaque fibre est nulle d'après une remarque ultérieure.

ThÉorÈme. Soit $N$ une variété différentiable munie d'une structure presquetransverse intégrable. On suppose que l'application : $N \rightarrow N / \mathcal{L}$ soit une submersion surjective. Si les fibres de la submersion sont connexes et simplement connexes et si la connexion plate induite sur chaque fibre est complète, alors $N$ est l'espace total d'un fibré vectoriel isomorphe au fibré transverse d'un feuilletage.

Preuve: On va montrer que la submersion $\pi: N \rightarrow M=N / \mathcal{L}$ est un fibré affine modelé sur le fibré transverse $(Q, p, M)$. Le théorème découle alors de la proposition 1 .

Soit $u \in Q_{x} ; u$ induit un champ de vecteurs $U$ sur $\pi^{-1}(x)$ dont la valeur en chaque point $y$ de $\pi^{-1}(x)$ est le relèvement vertical $u^{v}$ de $u$. On a $\nabla_{U} U=0$ d'après la proposition 3. Le champ géodésique $U$ engendre donc un groupe global de transformation à un paramètre $\left\{\gamma_{t}^{u}\right\}_{t \in \mathbb{R}}$. Pour $\forall y \in \pi^{-1}(x)$, l'application $t \rightarrow \gamma_{t}^{u}(y)$ est une courbe intégrale de $U$ telle que $\gamma_{0}^{u}(y)=y$. On pose :

$$
\rho_{x}(y, u)=\gamma_{1}^{u}(y)
$$

Soient $u, v \in Q_{x}$; ils définissent sur $\pi^{-1}(x)$ deux champs de vecteurs $U$ et $V$ tels que $[U, V]=0$. Par suite, leurs groupes globaux de transformation à un paramètre $\left\{\gamma_{v}^{u}\right\}_{t \in R}$ et $\left\{\gamma_{t}^{v}\right\}_{t \in \mathbb{R}}$ commutent. Il en résulte que le groupe global de transformations à un paramètre $\left\{\gamma_{t}^{u} \circ \gamma_{t}^{v}\right\}_{t \in \mathbb{R}}$ engendre le champ vertical défini par $u+v$. Donc

$$
\gamma_{t}^{u} \circ \gamma_{t}^{v}=\gamma_{t}^{v} \circ \gamma_{t}^{u}=\gamma_{t}^{u+v}
$$

Par suite

$$
\rho_{x}\left(\rho_{x}(y, u), v\right)=\rho_{x}(y, u+v) .
$$

Ainsi $Q_{x}$ opère sur $\pi^{-1}(x)$. Soit $(u, v)$ un produit scalaire sur $Q_{x}$. On obtient une métrique riemannienne $g$ sur $\pi^{-1}(x)$ en posant $g(U, V)=(u, v)$. Puisque $\nabla U=\nabla V=0$, on a $\nabla g=0$ et $\nabla$ est la connexion de Levi-Civita de la métrique $g$. Ainsi $\left(\pi^{-1}(x), g\right)$ est une variété riemannienne complète et connexe. Soit $y$ et $z$ 
deux éléments de $\pi^{-1}(x)$. Il existe une géodésique $c$ qui joint $y$ à $z$. On peut prendre $y=c(0)$ et en modifiant éventuellement $c(0)$, on peut supposer que $z=c(1)$. Or $c(0)$ est le relèvement vertical d'un élément $u$ de $Q_{x}$. Ainsi $c$ est la courbe intégrale $t \rightarrow \gamma_{t}^{u}(y)$ de $U$ passant par $y$. Donc $\gamma_{1}^{u}(y)=z=\rho_{x}(y, u)$ et l'action de $Q_{x}$ sur $\pi^{-1}(x)$ est surjective. Soit $H(y)$ le groupe d'isotropie de $y$ sous l'action de $Q_{x}$. $H_{y}=\left\{u \in Q_{x}, \rho_{x}(y, u)=y\right\}$. L'application de $Q_{x}$ dans $\pi^{-1}(x)$ qui à $u$ fait correspondre $\rho_{x}(y, u)$ est la composition d'un isomorphisme de $Q_{x}$ sur $T_{y}\left(\pi^{-1}(x)\right)$ et de l'application exponentielle de $\nabla$ qui est un difféomorphisme local. Donc $H(y)$ est un sous-groupe discret de $Q_{\boldsymbol{x}}$. Si $H(y)$ n'est pas trivial, il existe des vecteurs linéairement indépendants de $Q_{x}$ tels que $H(y)$ est l'ensemble des combinaisons à coefficients entiers de ces vecteurs. Comme $Q_{x}$ opère transitivement sur $\pi^{-1}(x)$, cette variété est difféomorphe à $\mathrm{T}^{k} \times \mathrm{R}^{p+q-k}$ où $\mathrm{T}^{k}$ est le tore de dimension $k$. Comme $\mathrm{T}^{k} \times \mathrm{R}^{p+q-k}$ n'est pas simplement connexe, nécessairement $H(y)=0$ et $Q_{x}$ opère librement sur $\pi^{-1}(x)$.

\section{REFERENCES}

[1] F. Brickell and R.S. Clark, 'Integrable almost tangent structures', J. Diff. Geom o (1974), $557-563$.

[2] M. Crampin and G. Thompson, 'Affine bundles and integrable almost tangent structures', Math. Pasc. Cambr. Phil. Soc. 98 (1985), 61-71.

[3] T.V. Dic, 'Structure presque-transverse', J. Diff. Geom. 14 (1979), 215-219.

[4] T.V. Duc, 'Structure presque tangente sur le fibré transverse', preprint.

[5] G. Thompson, 'Integrable almost cotangents structures and legendrian bundles', Math. Proc. Cambr. Phil. Soc. 101 (1987), 61-78.

Laboratoire de Mathématiques

Institute Fourier

Université de Grenoble I

B.P. 74

38402 Saint-Martin d'Heres

Cedex

France. 